\title{
ON THE SYMMETRIC PRODUCT OF A RATIONAL SURFACE
}

\section{ARTHUR MATTUCK ${ }^{1}$}

In his work on rational equivalence [5] Severi often raised this question: if the points of a nonsingular algebraic variety $V$ are all rationally equivalent to each other, is $V$ a unirational variety?

A variety $V$ is said to be unirational (over some field $k$ ) if it is the image of a projective space $P$ under a generically surjective rational map $s: P \rightarrow V$ which is defined over $k$, of finite degree, and separable. If $V$ is unirational, it is easily seen that all its points are rationally equivalent; Severi's question asks whether the converse is true. ${ }^{2}$ Now if it is when $V$ is a surface, an easy but interesting consequence would be the following theorem for which we will offer a direct proof. We work always over an algebraically closed field $k$.

THEOREM. Let $V$ be a surface over $k$ and let $V(n)$ denote its $n$-fold symmetric product. If $V(n)$ is unirational for some $n$, then $V$ is a rational surface.

Before proving this, we comment on a few aspects. The theorem has birational character, so we may assume $V$ is a nonsingular projective surface. If $V(n)$ is unirational, any two points are rationally equivalent and therefore any two positive 0 -cycles of degree $n$ on $V$ are rationally equivalent. It follows easily that any two points of $V$ are rationally equivalent and then an affirmative answer to Severi's question would imply that $V$ is unirational. This reduces us to the case $n=1$, in which case the result is a well-known consequence of the Castelnuovo-Zariski criterion for rationality (see below).

In another direction, it is classical that if $C$ is a curve, the map $C(n) \rightarrow J$ of the symmetric product onto the Jacobian has rational varieties for its fibers. The theorem shows this cannot be true for the corresponding map $V(n) \rightarrow A$ onto the Albanese variety (as has occasionally been conjectured), since a surface having trivial Albanese variety need not be rational.

Received by the editors May 8, 1968.

1 This research was supported in part by the National Science Foundation.

${ }^{2}$ ADDED IN PROOF. It now appears that the question has an affirmative answer for surfaces in characteristic zero. It follows from an easy generalization of techniques used by Mumford in a recent proof (to appear in the Kyoto Journal of Mathematics) that the rational equivalence ring is not finitely generated. 
The converse statement, $V$ rational $\Rightarrow V(n)$ unirational, is trivial. But $V$ rational $\Rightarrow V(n)$ rational is also true and for a variety $V$ of any dimension [3]. Thus if $V$ is a surface, we get $V(n)$ unirational $\Rightarrow V(n)$ rational. No example of a unirational variety which is not rational is yet known.

Finally, we note that the theorem definitely requires $k$ to be an algebraically closed ground field, even if the stronger hypothesis: $V(n)$ rational-is imposed. Even for $V$ a curve, it would otherwise be false. A conic $C$ over $k$ but without a $k$-point is not $k$-isomorphic to a projective line, yet $C(2)$ is $k$-isomorphic to the projective plane via the map which assigns to a pair of points on $C$ the line through them.

ProOF OF THE THEOREM. We are assuming $V(n)$ is unirational and may also assume $V$ is complete and nonsingular. As a first remark,

$$
V \text { has trivial Albanese variety. }
$$

For if $X$ is any variety, $\mathrm{Alb}[X(n)]=\mathrm{Alb}[X]$ by an elementary property of the Albanese variety; but since $V(n)$ is the rational image of $P$, there is an induced surjective map from $\mathrm{Alb}[P]$ to $\mathrm{Alb}[V(n)]$. Since $\operatorname{Alb}[P]=0$, the result (1) follows.

We denote as usual by $p_{a}$ the (classical) arithmetic genus of $V$, by $p_{g}=\operatorname{dim} H^{0}\left(V, \Omega_{2}\right)$ the geometric genus, by $P_{2}=\operatorname{dim} H^{0}\left(V, \Omega_{2} \otimes \Omega_{2}\right)$ the second plurigenus. Then with the given hypotheses,

$$
P_{2}(V)=0 \Rightarrow V \text { is a rational surface. }
$$

For by a classical relation $p_{a}-p_{a}=q=\operatorname{dim} \operatorname{Alb}(V)$. This is true in characteristic 0 , and by a theorem of Nakai (see [4]) also in characteristic $p$ provided that $p_{\theta}=0$. In our case, $p_{\theta}=0$ because clearly in general, $p_{\theta}>0 \Rightarrow P_{2}>0$. Thus from the relation and (1) we get $p_{a}=0$. By the Castelnuovo-Zariski criterion [6], [7] if $P_{2}=0$ and $p_{a}=0$, then $V$ is a rational surface.

$$
\text { If } V \text { is a complete nonsingular surface and } P_{2}(V)>0 \text {, }
$$

then $V(n)$ is not unirational for any $n$.

This will complete the proof of our theorem.

We begin with a few remarks about $m$-forms of weight $r$ on an $m$-dimensional variety $X$. These form a one-dimensional $k(X)$-space, since each has a unique representation in the form $g\left(d x_{1} \cdots d x_{m}\right)^{r}$, where $g \in k(X)$ and $x_{1}, \cdots, x_{m}$ is a separating transcendence base for $k(X) / k$. If $y_{1}, \cdots, y_{m}$ is another such basis, then the corresponding expressions are related by

$$
g\left(d x_{1} \cdots d x_{m}\right)^{r}=g\left(\frac{\partial\left(x_{1}, \cdots, x_{m}\right)}{\partial\left(y_{1}, \cdots, y_{m}\right)}\right)^{r}\left(d y_{1} \cdots d y_{m}\right)^{r} .
$$


If $X$ is complete and nonsingular, then the form (4) is by definition holomorphic at $p \in X$ if $g$ is holomorphic at $p$ when the $\left(x_{i}\right)$ are chosen to be uniformizing coordinates at $p$. The global holomorphic forms (4) may be identified with the sections of the $r$ th tensor power of the sheaf $\Omega_{m}$ of holomorphic $m$-forms. Thus they form a $k$-space of dimension $P_{r}(X)$, where $P_{r}(X) \equiv \operatorname{dim} H^{0}\left(X, \Omega_{m}^{\otimes_{r}}\right), r>0$.

We now proceed to the proof of (3). Let $x, y$ be a separating transcendence basis for $k(V) / k$. Since $P_{2}(V)>0$, there will be a nonzero holomorphic 2 -form of weight 2 on $V$,

$$
\phi=g(d x d y)^{2}, \quad g \in k(V) .
$$

Let $V[n]$ be the $n$-fold product of $V$, let $\pi_{i}: V[n] \rightarrow V$ be the projection maps, and $x_{i}=\pi_{i}^{*} x, \phi_{i}=g_{i}\left(d x_{i} d y_{i}\right)$, etc. Consider the $2 n$-form of weight 2 on $V[n]$ defined by

$$
\Phi=g_{1} g_{2} \cdots g_{n}\left(d x_{1} d y_{1} \cdots d x_{n} d y_{n}\right)^{2} .
$$

It is easily seen, using (4), that $\Phi$ is holomorphic on $V[n]$ and is well defined by $\phi$, that is, it does not depend on the choice of $x, y$. It is the existence of this form $\Phi$ which will show that $V(n)$ cannot be unirational. First of all, we claim that

$$
\Phi \text { is the lifting, via } V[n] \rightarrow V(n) \text {, of a form } \Phi_{0} \text { on } V(n) \text {. }
$$

Namely, choose a separating transcendence basis $u_{1}, \cdots, u_{2 n}$ for $k(V(n)) / k$ and write $\Phi=h\left(d u_{1} \cdots d u_{2 n}\right)^{2}$, where $h \in k(V[n])$. Since $\Phi$ and the $u_{i}$ are invariant under the $n$ ! automorphisms comprising the Galois group of $k(V[n]) / k(V(n))$, it follows from the uniqueness of the above representation (once the $u_{i}$ are chosen) that $h$ is also invariant, so $h \in k(V(n))$. This proves (6).

If $V(n)$ were nonsingular (which it is not) and $\Phi_{0}$ were holomorphic on $V(n)$, the proof of (3) would be concluded by the

Proposition. Let $X$ be a complete nonsingular variety of dimension $m>0$. If $X$ is unirational, then $P_{r}(X)=0$ for $r>0$.

Proof. If $X$ is projective space, $\Omega_{m}=\mathcal{O}(-m-1)$ and so $H^{0}\left(X, \Omega_{m}^{\otimes_{r}}\right)$ $=H^{0}(X, \mathcal{O}(-r m-r))$, and this is 0 for $r, m>0$. In the general case we use the separable, rational, and generically surjective map $s: P \rightarrow X$. Its fundamental locus on $P$ has codimension $\geqq 2$, by general principles. If $\omega$ is a holomorphic $m$-form of weight $r$ on $X$, then $s^{*} \omega$ is an $m$-form of weight $r$ on $P$ which is holomorphic except perhaps on a locus of codimension $\geqq 2$. Since any differential on a nonsingular variety always has divisors as its singularities, $s^{*} \omega$ must be holomorphic everywhere on $P$. Therefore by the first case of the proposition, $s^{*} \omega=0$, but since $s$ is separable, we get $\omega=0$ also. Thus $P_{r}(X)=0$. 
We cannot apply the proposition directly to $V(n)$ since it has singularities, even though $V$ does not. Fortunately however a canonical resolution of the singularities exists, valid in all characteristics. This is the Hilbert scheme $H_{n}(V)$, a $2 n$-dimensional variety whose points represent in a natural way all 0 -dimensional subschemes of $V$ having Hilbert polynomial $n$ (that is, defined by a sheaf of ideals $g \subset \Theta_{V}$ for which $\operatorname{dim}_{k}(\theta / g)=n$ ). (For the facts about $H_{n}$ used here, see Fogarty [1] and [2].) The variety $H_{n}$ is complete, irreducible and nonsingular, and there is a birational morphism

$$
f: H_{n} \rightarrow V(n) \text {. }
$$

We will now prove that

$$
f^{*} \Phi_{0} \text { is holomorphic on } H_{n} \text {. }
$$

This will complete the proof of (3) and thus of the theorem, for according to the proposition it shows that $H_{n}$ is not unirational, and therefore neither is $V(n)$ since the morphism (7) is birational.

Let $S$ denote the singular locus of $V(n)$. Clearly the poles of $f^{*} \Phi_{0}$ have to lie in $f^{-1}(S)$. But $f^{-1}(S)$ is known ${ }^{3}$ to have only one divisorial component $D$, so to prove (8) it suffices to show $f^{*} \Phi_{0}$ is holomorphic at a general point $q$ of $D$. Now such a point $q$ represents a subscheme of $V$ having the form $Z \cup p_{3} \cup \ldots \cup p_{n}$, where $p_{i} \neq p_{j}$ and where $Z$ is a subscheme of length 2 concentrated over a point $p \neq p_{i}$. This in effect permits a reduction of the theorem to the case where $n=2$. For suppose $z \in H_{2}(V)$ represents $Z$. We have

$$
H_{2} \times V[n-2] \rightarrow H_{n}
$$

a rational map defined in an obvious way which is a finite morphism in a neighborhood of $\left(z, p_{3}, \cdots, p_{n}\right)$. Let $x, y$ be uniformizing coordinates at all the points $p, p_{3}, \cdots, p_{n}$ and let $u_{1}, \cdots, u_{4}$ be uniformizing coordinates at $z$. Then $f^{*} \Phi_{0}$ lifts via the map (9) to a differential $\Phi_{0}^{*}$ which in view of (4) and (5) may be written

${ }^{3}$ Added in page proof. This statement unfortunately turns out to be not known, but only conjectured. Thus while the ensuing calculation is of interest, it is not suffcient for proving (8). Instead prove (8) this way. Let $W$ be the normalization of $H_{n}$ in the function field of $V[n]$. If $f^{*} \Phi_{0}$ were not holomorphic on $H_{n}$, it would have a divisorial pole, and so therefore would its lifting to $W$. But since $\Phi$ is holomorphic on the nonsingular variety $V[n]$, it cannot have poles on any normal variety birationally equivalent to $V[n]$, such as $W$.

Note also that a generalization of work of Mumford [Rational equivalence of zerocycles, Kyoto J. Math. (to appear) ] will give an affirmative answer to Severi's question in characteristic 0 . The present paper is valid in arbitrary characteristic, of course. 


$$
\Phi_{0}^{*}=g_{1} g_{2} \cdots g_{n}\left(\frac{\partial\left(x_{1}, y_{1}, x_{2}, y_{2}\right)}{\partial\left(u_{1}, u_{2}, u_{3}, u_{4}\right)}\right)^{2}\left(d u_{1} \cdots d u_{4} d x_{3} \cdots d y_{n}\right)^{2}
$$

and it suffices to prove $\Phi_{0}^{*}$ is holomorphic at $\left(z, p_{3}, \cdots, p_{n}\right)$. Now certainly $g_{3}, \cdots, g_{n}$ are holomorphic there. Since $g_{1} g_{2}$, viewed as a function on $V[2]$, is holomorphic at $(p, p)$ and symmetric, it can be viewed as a function on $V(2)$ and is holomorphic at the point of $V(2)$ corresponding to $2 p$. Thus it is holomorphic at $z \in H_{2}$ as well since the map (7) is a morphism.

Thus we are reduced to showing that the Jacobian in (10), viewed as a function on $\mathrm{H}_{2}$, is holomorphic at the point $z$. The functions $x, y$ in $k(V)$ define a rational map $h: V \rightarrow A$ of $V$ into the affine plane $A$. Let $W$ be any dominating desingularization of the symmetric product $A(2)$. Then we get a diagram

$$
\begin{array}{rrr}
V & V(2) \underset{f_{2}}{\leftarrow} H_{2} \\
h \downarrow & h_{1} \downarrow & \downarrow h_{2} \\
A & A(2) \underset{f_{1}}{\leftarrow} W
\end{array}
$$

where the vertical maps $h_{1}$ and $h_{2}$ are separable, rational, and generically surjective, while the horizontal ones are birational morphisms.

The differential $\Psi=d x_{1} d y_{1} d x_{2} d y_{2}$ on $V[2]$ is the lifting of a differential on $A(2)$ which we denote by $\Psi$. At the point $z \in H_{2}$,

$$
\left(f_{2} h_{1}\right)^{*} \Psi=\frac{\partial\left(x_{1}, y_{1}, x_{2}, y_{2}\right)}{\partial\left(u_{1}, u_{2}, u_{3}, u_{4}\right)} d u_{1} d u_{2} d u_{3} d u_{4}
$$

Showing the Jacobian in (10) is holomorphic at $z$ is the same as showing that this differential is holomorphic at $z$. Since $h_{2}$ is holomorphic at $z$, it being the general point of a divisor on the nonsingular variety $\mathrm{H}_{2}$, it suffices to prove that

$$
f_{1}^{*} \Psi \text { is holomorphic at } h_{2}(z) \text {, for some } W \text {. }
$$

We choose as $W$ the monoidal transform of $A(2)$ along its singular locus and prove (11) by explicit computation. We have as coordinates in $A$ [2] the set $\left(x_{1}, y_{1}, x_{2}, y_{2}\right)$, and the subring of the coordinate ring which is invariant under the interchange of $x_{1}, y_{1}$ with the pair $x_{2}, y_{2}$ is generated by the five polynomials

$$
z_{1}=x_{1}+x_{2}, \quad z_{2}=y_{1}+y_{2}, \quad z_{3}=x_{1} x_{2}, \quad z_{4}=y_{1} y_{2}, \quad z_{5}=x_{2} y_{1}+x_{1} y_{2} .
$$

Assuming first that the characteristic is not 2, a more convenient set of generators is given by the following polynomials in $z_{1}, \cdots, z_{5}$ :

$$
z_{1}, z_{2}, t=\left(x_{1}-x_{2}\right)\left(y_{1}-y_{2}\right), \quad u=\left(x_{1}-x_{2}\right)^{2}, \quad v=\left(y_{1}-y_{2}\right)^{2} \text {. }
$$


Then $A(2)$ is given as a hypersurface in affine 5 -space by the equation $t^{2}=u v$. This shows it is the product of a cone $C$ with a plane. The singular locus $S_{0}$ is where $\left(x_{1}, y_{1}\right)=\left(x_{2}, y_{2}\right)$, i.e., where $t=u=v=0$. The monoidal transform $W$ of $A(2)$ along $S_{0}$ is $W=C^{\prime} \times A$ where $C^{\prime}$ is the quadratic transform of the cone $C$, well known to be nonsingular. Thus $W$ is nonsingular also. Coordinates for an open set in $W$ are $\left(z_{1}, z_{2}, t, u^{\prime}, v^{\prime}\right)$ where $u^{\prime}=u / t, v^{\prime}=v / t$, the equation being $u^{\prime} v^{\prime}-1=0$. At a general point $p_{0}$ of the divisor $D_{0}$ on $W$ which is the inverse image of $S_{0}$, the function $u^{\prime}=\left(x_{1}-x_{2}\right) /\left(y_{1}-y_{2}\right)$ does not vanish. Therefore $z_{1}, z_{2}, t, u^{\prime}$ are uniformizing coordinates at $p_{0}$ (since $\partial_{v^{\prime}}\left(u^{\prime} v^{\prime}-1\right) \neq 0$ at $\left.p_{0}\right)$ and in terms of these one can check that $\Psi$ is holomorphic at $p_{0}$, for we have $\Psi=d x_{1} d y_{1} d x_{2} d y_{2}=-\left(1 / 8 u^{\prime}\right) d z_{1} d z_{2} d t d u^{\prime}$.

If the characteristic is 2 , the calculation is similar. Using the original coordinates, the equation of $A(2)$ is

$$
z_{5}\left(z_{5}-z_{1} z_{2}\right)+z_{3} z_{2}^{2}+z_{4} z_{1}^{2}=0 .
$$

The singular locus is $z_{1}=z_{2}=z_{5}=0$. A typical open affine set of the monoidal transform would be given by the coordinates $\left(z_{1}{ }^{\prime}, z_{2}{ }^{\prime}, z_{3}, z_{4}, z_{5}\right)$ where $z_{1}^{\prime}=z_{1} / z_{5}, \quad z_{2}^{\prime}=z_{2} / z_{5}$ and the equation is seen to be $1+z_{1}^{\prime} z_{2}^{\prime} z_{5}+z_{3} z_{2}^{\prime 2}+z_{4} z^{\prime 2}{ }_{1}=0$. This is nonsingular. Similarly the other open affines which cover $W$ are nonsingular. At a general point $p_{0}$ of $D_{0}$, we have $z_{5}=0, z_{1}{ }^{\prime} \neq 0, z_{2}{ }^{\prime} \neq 0$ (since $z_{3}$ and $z_{4}$ have generic values). Thus $z_{1}{ }^{\prime}, z_{2}{ }^{\prime}, z_{3}, z_{4}$ are uniformizing coordinates at $p_{0}$ and once again we can check that $\Psi$ is holomorphic at $p_{0}$, for

$$
\Psi=d x_{1} d y_{1} d x_{2} d y_{2}=\frac{1}{z_{1} z_{2}} d z_{1} d z_{2} d z_{3} d z_{4}=\frac{1}{{z_{1}{ }^{\prime} z_{2}{ }^{\prime}}^{\prime}} d z_{1}{ }^{\prime} d z_{2}{ }^{\prime} d z_{3} d z_{4} .
$$

\section{BiBLIOGRAPHY}

1. J. Fogarty, On Hilbert schemes, Dissertation, Harvard Univ., Cambridge, Mass., 1966.

2. - - Algebraic families on an algebraic surface, Amer. J. Math. 90 (1968), 511-521.

3. A. Mattuck, On the field of multisymmetric functions, Proc. Amer. Math. Soc. 19 (1968), 764-765.

4. D. Mumford, Lectures on curves on an algebraic surface, Princeton Univ. Press, Princeton, N. J., 1965; pp. 198-200 (ref. [24]).

5. F. Severi, Problèmes résolus et problèmes nouveaux dans la théorie des systemes d'équivalence, Proc. Internat. Cong. Math. 1954, Amsterdam, Vol. 3, p. 539, NorthHolland, Amsterdam, 1956.

6. O. Zariski, The problem of minimal models in the theory of algebraic surfaces, Amer. J. Math. 80 (1958), 146-184.

7. - On Castelnuovo's criterion of rationality of an algebraic surface, Illinois J. Math. 2 (1958), 303-315.

Massachusetts Institute of Technology 